\title{
Verzeichnis der Abkürzungen
}

1. Die $\S \S$ des BGB. sind nur mit der Zahl zitiert. Bei allen übrigen Gesetzen erfolgt das Zitat unter abgekürzter Angabe der Gesetzesstelle, etwa: EG. $2=$ Einführungsgesetz zum BGB. Art. 2.

2. Auf andere Stellen dieses Buches wird durch den Vorsatz „oben" oder „unten“" hingewiesen.

3. Einzelne Abkürzungen.

AcP. $\quad=$ Archiv für zivilistische Praxis.

AG. $\quad=$ Ausführungsgesetz zum BGB.

AktG. $\quad=$ Aktiengesetz vom 30. Januar 1937.

ALR. $\quad=$ Preußisches Allgemeines Landrecht.

ArchöffR. = Archiv für öffentliches Recht.

AV. $\quad=$ Ausführungsverordnung.

BGB. = Bürgerliches Gesetzbuch für das Deutsche Reich.

BelZG. $\quad=$ Preußisches Gesetz über den Belagerungszustand vom 4.6.1851.

Bensh. $\quad=$ Entscheidungen des Reichsarbeitsgerichts und der Landesarbeitsgerichte, Verlag Bensheimer.

BGH. = Entscheidungen des Bundesgerichtshofes in Zivilsachen, seit 1951.

BRV. $\quad=$ Bundesratsverordnung.

BürgA. = Archiv für bürgerliches Recht.

DDR. = Deutsche Demokratische Republik.

Denkschr. = Denkschrift zum BGB.

DJZ. $\quad=$ Deutsche Juristenzeitung.

DR.

$\begin{array}{ll}\text { DRZsch. } & =\text { Deutsche Rechts-Zeitschrift. Herausgegeben von Karl S. Bader, }\end{array}$ Freiburg i. Br.; Verlag J. C. B. Mohr, Tübingen.

DogmJ. $\quad=$ Jherings Jahrbücher für die Dogmatik des heutigen römischen nnd deutschen Privatrechts, jetzt des bürgerlichen Rechts.

EG. $\quad=$ Einführungsgesetz.

EGBGB. = Einführungsgesetz zum Bürgerlichen Gesetzbuch vom 18.3.1896.

FrGG. $\quad=$ Reichsgesetz über die Angelegenheiten der freiwilligen Gerichtsbarkeit vom 17. 5. 1898.

G. $\quad=$ Gesetz.

GG. = Grundgesetz für die Bundesrepublik Deutschland v. 23. 5. 1949.

GBO. $=$ Grundbuchordnung.

Gew0. = Gewerbeordnung für das Deutsche Reich.

Gruchot = Beitr. zur Erläuterung des deutschen Rechts, begründet von J. Gruchot.

Grünhut = Zeitschrift für das Privat- und öffentliche Recht von Grünhut.

GVG. $\quad=$ Gerichtsverfassungsgesetz für das Deutsche Reich vom 27.1.1877, 22. 3. 1924 .

HaftpflG. = Gesetz betr. die Verbindlichkeit zum Schadenersatz für die bei dem Betriebe von Eisenbahnen, Bergwerken usw. herbeigeführten Tötungen und Körperverletzungen vom 7.6.1871.

HdwbR. $=$ Handwörterbuch der Rechtswissenschaft.

HGB. $\quad=$ Handelsgesetzbuch vom 10.

HEZ. $\quad=$ Höchstrichterliche Entscheidungen in Zivilsachen, seit 1948. 
JRdsch. $\quad=$ Juristische Rundschau, seit 1925.

JW. $\quad=$ Juristische Wochenschrift.

JZ. $=$ Juristenzeitung, seit 1951.

KO. = Konkursordnung.

KrfG. $\quad=$ Kraftfahrzeuggesetz.

KunstUrhG. = Gesetz betr. das Urheberrecht an Werken der bildenden Künste und der Photographie vom 9.1.1907/22. 5. 1910.

LitUrhG. = Gesetz betr. das Urheberrecht an Werken der Literatur und der Tonkunst vom 19.6.1901/22. 5. 1910.

LZ. $\quad=$ Leipziger Zeitschrift für deutsches Recht.

Mot. = Motive zum Entwurf eines BGB. (amtliche Ausgabe).

NJW. $\quad=$ Neue Juristische Wochenschrift seit 1947.

OGHbrZ. = Entscheidungen des Obersten Gerichtshofes für die brit. Zone in Zivilsachen von 1948-1950.

OLG. = Rechtsprechung der Oberlandesgerichte, herausgegeben von Mugdan und Falkmann.

PStG. $\quad=$ Personenstandsgesetz vom 3. 11. 1937/15. 1. 1951.

PostG. $\quad=$ Gesetz über das Postwesen des Deutschen Reiches vom 28.10. 1871/20. Dezember 1899/5. Februar 1925/13. 12. 1933.

PrV. = Preußische Verfassungsurkunde vom 30.11.1920.

Prot. $\quad=$ Protokolle der Kommission für die II. Lesung des Entwurfs des BGB., bearbeitet von Achilles u. a.

Recht = Das Recht, Rundschau für den deutschen Juristenstand.

RdA. $\quad=$ Recht der Arbeit.

RG. = Entscheidungen des Reichsgerichts in Zivilsachen.

RGRKomm. = Kommentar von Reichsgerichtsräten zum BGB.

RGStr. = Entscheidungen des Reichsgerichts in Strafsachen.

RGWarn. = Warneyer, Rechtsprechung des Reichsgerichts.

RV. $\quad=$ Gesetz betr. Verfassung des Deutschen Reiches vom 16. 4. 1871.

RVerf. oder WeimV. = Verfassung des Deutschen Reiches vom 11.8.1919.

RvglHdwb. = Rechtsvergleichendes Handwörterbuch für das Zivil- und Handelsrecht des In- und Auslandes.

RVerG. = Reichsvereinsgesetz vom 19.4. 1908.

RVO. $\quad=$ Reichsversicherungsordnung.

SeuffA. $\quad=$ Seufferts Archiv für Entscheidungen der obersten Gerichte.

SeuffBl. = Seufferts Blätter für Rechtsanwendung.

StGB. $\quad=$ Strafgesetzbuch für das Deutsche Reich vom 15.5.1871.

SüddJZ. = Süddeutsche Juristenzeitung. Herausgegeben von Geiler u. a. Verlag Lambert Schneider, Heidelberg.

UnlWG. $\quad=$ Gesetz zur Bekämpfung des unlauteren Wettbewerbs vom 27.5. $1896,7.6 .1909$.

vo. $\quad=$ Verordnung.

WarenzeichenG. = Gesetz zum Schutze der Warenbezeichnungen vom 5. 5.1936.

Warn. $\quad=$ Warneyer, Jahrbuch der Entscheidungen.

WG. $\quad=$ Wechselgesetz vom 21.6.1933.

ZfHR. = Zeitschrift für das gesamte Handelsrecht.

ZentrBlfHR. = Zentralblatt für Handelsrecht.

ZivA. = Archiv für zivilistische Praxis.

ZPO. $\quad$ Z ZivilprozeBordnung.

ZVG. $\quad=$ Gesetz über die Zwangsversteigerung und Zwangsverwaltung rom 24. 3. 1897. 
